\title{
Special characteristics of multi-product manufacturing
}

\author{
Sergey Egorov ${ }^{1}$, Alexey Kapitanov ${ }^{2}$, Vladimir Mitrofanov ${ }^{3}$ and Tatiana Egorova ${ }^{4}$ \\ ${ }_{1,2,3,4}$ Moscow state university of technology "STANKIN"
}

\begin{abstract}
The article considers the relevant processes of transition from "traditional" types of computeraided manufacturing, including both large-scale and mass production, to flexible multi-product manufacturing. In this case, the mission to produce different new part lots is allotted to manufacturing systems consisting of machines with rather wide technological capabilities.

In this article, the authors propose an original solution to one of the problems specifying the multi-product manufacturing - a rational choice of equipment capable to produce a given range of products in computeraided manufacturing. The authors analyzed special characteristics of multi-product manufacturing, as well as identified and justified the need for elaborating a method for classification of workpieces and choice of machines for their processing. According to this study, the authors propose a method for developing the technology for manufacturing multi-products parts $[5,6]$.
\end{abstract}

Keywords: multi-product manufacturing, flexible manufacturing system, computer-aided manufacturing

\section{Introduction}

The constantly changing market conditions lead to an increase in the production of a wide range of products in small batches. According to the International Academy for Production Engineering (CIRP), even nowadays the share of medium and small-scale manufacturing exceeds $70 \%$ worldwide. A steady trend of moving from mass and large-scale production to small-scale and unit production is observed even in manufacturing fields, such as for example, automobile industry traditionally producing large scale goods. It requires the usage of all reserves available to the traditional forms of organization.

Modern companies have to operate in highly variable and dynamic environment that requires flexibility and adaptability to constantly changing market conditions, and in order to effectively change the established production plans, some appropriate technologies should be introduced to manufacturing fields. Flexible manufacturing systems (FMS) are considered nowadays as a model of implementation of modern computeraided technologies and devices within the concept of computer-aided manufacturing and wider use of industrial robots and manipulating systems [1,2,3]. A quick-change-over capability of production to the needs of different customers is becoming an increasingly important factor in international market competition; therefore, the development of flexible manufacturing systems is an important success criterion, together with respective organizational decisions.

\section{Main part}

One of the main tasks to be solved at the FMS design stage is to identify types of machines for computer-aided manufacturing (CAM). Initial data for the selection of a computer-aided machine subsystem are the information database of machines and manufactural parts.

The classification of parts makes it possible to define by certain characteristics some part groups $\left(P_{i}^{*}\right)$, the processing of which will be most efficient in a manufacturing system.

\footnotetext{
${ }^{+}$Corresponding author. Tel.: +7 (925) 5047305.

E-mail address: egorovsergey@yandex.ru.
} 
In general, when designing FMS (computer-aided machine subsystem), we deal with a lot of machines $(O)$ and parts $(P)$.

As we know, not every part can be processed on any machine. The processing is possible in cases when the technological capabilities of the machine meet the process requirements of the part. The degree of such conformity can be measured by a coefficient $K_{o p}$.

$$
K_{o p}=\frac{\left|f_{o} \cap f_{p}\right|}{f_{p}}
$$

where $f_{o}$ - number of technological functions performed by a machine;

$f_{p}$ - number of technological functions required for complete part process.

When processing a part outside CAM it is desirable that $K_{o p}$ coefficient equals to 1 (the part is completely processed on this machine).

If parts are produced on CAM, not only 1 part is of main interest, but set of parts $\left(P^{*}\right)$ selected for processing in the system, thus it is required to consider $K_{o p}$ not only for some particular machine and part, but for CAM and set of parts ( $\left.\mathrm{P}^{*}\right)$. In this case, $K_{o p_{E S P}}$ is determined by the following formula:

$$
K_{o p_{E S P}}=\frac{\left|f_{o_{E S P}} \cap f_{p^{*}}\right|}{\left|f_{p^{*}}\right|},
$$

where $f_{{ }_{E S P}}$ - number of technological functions performed by a machine subsystem;

$f_{p} *-$ number of technological functions required for complete part process $\left(\mathrm{P}^{*}\right)$.

If possible one should choose a machine subsystem in such a way that $f_{p} * \subseteq f_{o_{F S P}}$, i. e. technological capabilities of the machine subsystem and process requirements for parts fully coincide $\left(K_{o p_{E S P}}=1\right)$.

To do this, machines from the information database should be sorted into groups $\left(O_{i: i=\overline{1, k}}^{*}\right.$, where $k$ is a machine group), which are characterized by the following similar parameters:

- table dimensions;

- control type;

- operating system;

- method of retooling (manual, automatic).

$O_{i: i=\overline{1, k}}^{*}=\left\langle p_{s_{1}}, p_{s_{2}}, p_{s_{3}}, p_{s_{4}}\right\rangle$.

In his analysis, the designer extracts more suitable machine (or machines) out of homogeneous groups (same size and type of table as well as technological capabilities) taking into account the following criteria:

- manufacturing company

- maintenance (its availability),

- purchase price;

- working cost per hour;

- tool slot capacity;

- accuracy class of the machine,

- positional accuracy of the machine,

- country of origin.

So, the selected machine group (machine groups) should process corresponding parts taking into account the conformity of overall dimensions of parts and machine tables as well as relative processing types of machine groups and relating parts. 
Subject to processing time and complexity of parts, the decision is made on what kind of machine group will be used in the designed CAM. This machine group is considered to single out possible variants of machine subsystems.

Generally, we deal with many types of parts

$$
R P=\left\{R P_{1}, R P_{2}, \ldots, R P_{i}\right\},
$$

where: $i$ - number of part types.

After the selection and choice have been made, we have a variety of different-type machines $O$ $=\left\{O_{1}, O_{2}, \ldots, O_{k}\right\}$. Each machine (machine type) has some technological capabilities (types of processing operations that can be performed on it).

$$
O_{i} \rightarrow R O\left(O_{i}\right)=\left\{R O_{1}, R O_{2}, \ldots, R O l\right\}
$$

where $l$-number of processing types possible to be performed on this machine

The cost of the machine $O_{i}$ equals to $K_{i}$

First of all, it is necessary to define a set of machines chosen from the variety of selected machines that can be used for all processing types required for producing a variety of parts. To solve the problem an algorithm of generation of possible variants is used (machine selector). In general, there can be a number of variants received. A considerable number of them will have greater technological capabilities rather than those required for machining, and therefore, will contain narrower variants (consisting of fewer machine types), but perfectly meeting process requirements. These variants (wider variants) will be immediately rejected. Variants that are not subset of others (wider variants) can be taken into account when selecting machine types of the CAM subsystem.

One or several variants are chosen among those for further analysis, if there are also a lot of them, taking into account the cost of machines and their technological characteristics. Thus, there is (for each variant) a great number of machine types required for processing a great many of parts.

$$
T O=\left\{T O_{1}, T O_{2}, \ldots, T O_{k}\right\}
$$

where $k$ - number of machine types.

In order to determine a machine subsystem, it is required to calculate the number of machines of each type. Each type of processing corresponds to the time required for its implementation.

$$
R O_{i} \rightarrow T_{i(i=1,2, \ldots, n)}
$$

Time fund of each machine run $O_{i}$ equals to $F$.

\section{Conclusion}

The task of determining the number of machines of each type is solved by a linear programming method.

A linear function, the minimum of which has to be found, is written as follows:

$$
K=\sum_{i=1}^{n} \sum_{j=1}^{m} C_{i} x_{i j} \rightarrow \min
$$

where: $K$ - cost of machine subsystem,

$C_{i}$ - cost of machine of the $i$ - type,

$x_{i j}$ - machine of the $i$-type implementing the $j$-processing type,

$n$ - number of machines in subsystem,

$m$-number of processing types required for part processing. Restrictions are stated as follows:

$$
\sum_{i=1}^{n} A_{i j} x_{i j} F=T_{j}, \mathrm{j}=1,2, \ldots, m, \quad x_{i j} \geq 0
$$


where $A_{i j}$-possibility to perform the $j$-type processing on the $i$-type machine;

$T_{j}$ - implementation type (for a great number of parts) of the $j$-type processing.

The above-stated task (linear programming) is solved by a simple method. On calculating the value $x_{i j}$, we can determine the number of machines of each type according to their technological capabilities.

$$
\sum_{j=1}^{m} x_{i j}=x_{i}, i=1,2, \ldots, n
$$

Thus, the solution obtained should be brought to integer form.

The decision on selecting a particular machine subsystem [3,7] is made on the cost-effectiveness analysis of processing a great number of parts in the following analyzed groups of machines. For this purpose, the part process is imitated in each of the selected machine subsystem. It allows determining the main characteristics of the system required for the selection of a rational subsystem of computer-aided machines.

\section{Acknowledgements}

The reported study was funded by RFBR, according to the research project No. 16-31-60079 mol_a_dk.

These results obtained in the framework of applied research conducted with the financial support of the Ministry of education of Russian Federation under the agreement No. 14.574.21.0127 from 28 November 2014 Unique identifier of the project RFMEFI57414X0127.

\section{References}

[1] S. Egorov, Technical youth education - centres technological support of additional education of children. Scientific Journal "Fundamental research", ISSN 1812-7339, 2014, No. 6, part. 5, p. 920-927.

[2] S. Egorov, Integrated educational and methodical complex for programming technology, cnc systems and development of control programs studies. Scientific Journal "Fundamental research", ISSN 1812-7339, 2014, No. 8, part. 1, p. 26-31.

[3] S. Egorov, Educational and methodical complex - a center of high-tech equipment with cnc and technological preparation of production. Scientific Journal "Modern problems of science and education", ISSN 2070-7428, 2014, No. 3, URL: www.science-education.ru/117-13240

[4] S. Egorov, Innovative educational-industrial complex on the basis of modern technological equipment with CNC and integrated system of production preparation in the field of machining. Journal «Vestnik «MSTU «STANKIN», ISSN 2072-3172, 2014, No. 3, p. 31-34.

[5] V.G. Mitrofanov, A.V. Kapitanov, A.P. Popov, Design of automated engineering industries. Irbit, Tolyatty: ONIKS, 2013 - 282 p. ISBN 978-5-9903090-8-1

[6] D. Loktev, S. Egorov, A. Kapitanov, V. Mitrofanov, T. Egorova, Modern means of measurement of shafts. STIN, 10, 2015, p. 13-19

[7] Egorov S.B., Kapitanov A.V., Loktev A.A., Loktev D.A., Mitrofanov V.G., Methods of checking the suitability of measurement processes for compliance with the requirements of the suitability for usw of modern metrological equipment. Journal «Vestnik «MSTU «STANKIN», ISSN 2072-3172, 2015, No. 4 (35), p. 83-88.

[8] Egorov S.B., Kapitanov A.V., Loktev D.A., Mitrofanov V.G., Egorova T.P., Ensuring the accuracy of measurement errors of form and position of surfaces on the basis of innovative equipment. Journal «Innovations», ISSN 2071-3010, № 8 (202), August 2015 